\title{
Comparison of spectral domain and swept source optical coherence tomography for angle assessment of Chinese elderly subjects
}

\author{
Yunsheng Qiao ${ }^{1}$, Chen $\operatorname{Tan}^{1}$, Min Zhang ${ }^{1,3}$, Xinghuai Sun ${ }^{1,2,3}$ and Junyi Chen ${ }^{1 *}$
}

\begin{abstract}
Background: This comparative study aimed to demonstrate the differences between swept source OCT (SS-OCT) $(1310 \mathrm{~nm})$ and spectral domain OCT (SD-OCT) $(840 \mathrm{~nm})$ for the identification and measurement of anterior chamber angle (ACA) structures.

Methods: Sixty seven eyes from 67 healthy subjects underwent ACA imaging at the nasal and temporal sides using SS-OCT and SD-OCT with different wavelength (Tomey, $1310 \mathrm{~nm}$ and RTVue, $840 \mathrm{~nm}$ ). Images were evaluated for the ability to distinguish angle structures including the Schwalbe's line (SL), the Schlemm's canal (SC) and the scleral spur (SS). The length of trabecular meshwork (LTM), the angle-opening distance (AOD500 and AOD750) and the length of Schlemm's canal (LSC) were also measured.

Results: The nasal identification rate for SL, SC and SS were 91.04\%/89.55\%, 50.75\%/40.30\% and 100.0\%/74.63\% (SSOCT/SD-OCT), respectively. The temporal identification rate for SL, SC and SS were 86.57\%/91.04\%, 68.66\%/70.15\% and $100.0 \% / 65.67 \%$ (SS-OCT/SD-OCT), respectively. Differences between SS-OCT and SD-OCT were found in terms of the visualization of the SS. With respect to the measurements of angle, the evaluation of LTM at the nasal side, LSC at the temporal side and AOD500/750 at both sides showed significant difference between the two devices. However, there existed good correlation between the AOD500/750 measured by SS-OCT and SD-OCT (Spearman's rank correlation coefficient $>0.8, p<0.000$ ).

Conclusions: SS-OCT displayed a better performance in detecting deeper structures of the angle such as the SS. However, for discriminating structures lying in transparent or semi-transparent tissue such as the SL and the SC, the two devices showed good consistency. Although SS-OCT and SD-OCT demonstrated high correlation for angle measurement (AOD500/750), their agreement was poor.
\end{abstract}

Keywords: Optical coherence tomography, Spectral domain, Swept source, Angle, Wavelength

\section{Background}

The assessment of the ACA is essential for diagnosis and treatment of glaucoma. Remaining the gold standard for the evaluation of ACA, the gonioscopy permits direct visualization of angle structures through microscopeaided eyes. However, the evaluation is relatively subjective and falls short of precision.

\footnotetext{
* Correspondence: chenjy@fudan.edu.cn

${ }^{1}$ Department of Ophthalmology \& Visual Science, Eye and ENT Hospital of

Fudan University, 83 Fenyang Rd, Shanghai 200031, China

Full list of author information is available at the end of the article
}

The introduction of ultrasound biomicroscopy (UBM) and anterior segment optical coherence tomography (AS-OCT) paved the way for precise angle measurement. Compared to UBM, AS-OCT provides noncontact, in vivo imaging of ACA together with other benefits such as higher axial resolution and shorter acquisition time [1, 2].

The application of Fourier-domain OCT (FD-OCT) was expanded in the field of angle assessment with higher resolution and scanning speed in contrast to time-domain OCT (TD-OCT). FD-OCT can be further

(c) The Author(s). 2019 Open Access This article is distributed under the terms of the Creative Commons Attribution 4.0 International License (http://creativecommons.org/licenses/by/4.0/), which permits unrestricted use, distribution, and 
divided into spectral-domain OCT (SD-OCT) and swept-source OCT (SS-OCT). SD-OCT devices use a broadband near-infrared superluminescent diode as the light source with a spectrometer as the detector. In this discussion, we chose RTVue (Optovue Corporation, Fremont, CA) with a central wavelength of around $840 \mathrm{~nm}$ [3]. On the other hand, SS-OCT instruments apply a tunable swept laser as the light source with a single photodiode detector. SS-1000 CASIA (Tomey, Nagoya, Japan) has been selected as the representative with the wavelength centered approximately $1310 \mathrm{~nm}$ [3]. The difference of operating mechanisms inevitably leads to the disparities of imaging quality and detecting capability between the two subtypes of FD-OCT which requires further demonstration and clarification. The purpose was to compare SD-OCT and SS-OCT concerning the identification and measurement of angle structures as well as evaluate the correlation and agreement between the two.

\section{Methods}

In this cross-sectional study, sixty-seven healthy Chinese elderly subjects were recruited for ACA evaluation from 11, November to 23, December, 2016 at the Eye and ENT Hospital of Fudan University, Shanghai, China. All subjects underwent a series of ocular evaluations including a detailed medical history taking, slit-lamp biomicroscopy, refraction examination, A-scan, ultrasound biomicroscopy, Goldmann applanation tonometry, gonioscopy, dilated fundus examination and standard automated perimetry. The inclusion criteria are: normal-appearing anterior segment, open ACA, intraocular pressure between 10 and $21 \mathrm{mmHg}$, normal fundus appearance and no sign of visual field defect. Subjects with best-corrected visual acuity of $\leq 20 / 40$, spherical refractive error $>+3$ or $<$ $-3 \mathrm{D}$, axial length $>25 \mathrm{~mm}$ or $<19 \mathrm{~mm}$, evidence of peripheral anterior synechiae on indentation by gonioscopy, previous use of any topical or systemic medication that could affect the aqueous humor circulation, history of intraocular surgery or penetrating trauma, laser trabeculoplasty, laser iridotomy, or laser iridoplasty were excluded from the study. When both eyes of the same subject were qualified, one eye was selected randomly.

\section{Anterior segment OCT imaging}

Anterior segment imaging was performed under dark conditions by a single trained examiner (JYC) masked to clinical findings. The two OCT devices were set in the same dark room. The order of examination was randomly decided. For each subject, a certain anatomical mark (e.g. a conjunctival vessel or pigmentation) was chosen to make sure that the same part of ACA was assessed and compared (Fig. 1 and Fig. 2). The external target light of each instruments was used to direct the

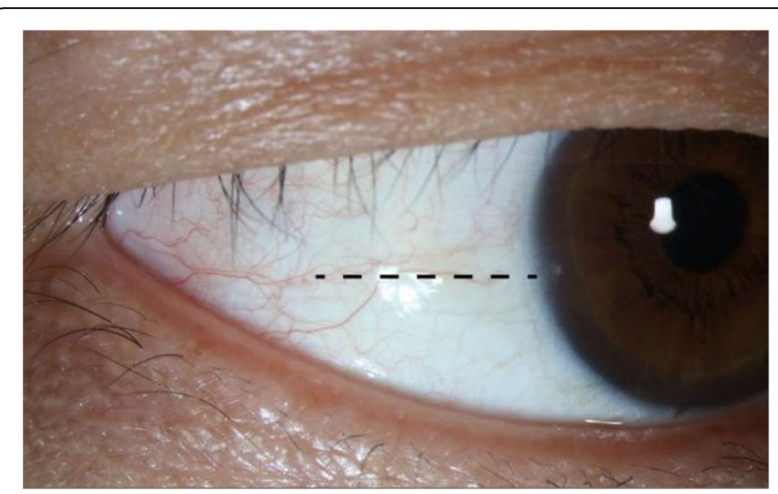

Fig. 1 A picture demonstrates the temporal corneal limbus. The black dotted line indicates the conjunctival vessel chosen as the anatomical landmark for OCT examination

patients' fixation. The brightness of the two fixation lights was measured by commercially available photometer [UT383 (UNI-T, Guangdong, China)] and no significance of statistical difference was found (Mean brightness for SS-OCT and SD-OCT were 75.0 and 77.4, $p$ value $=0.334)$. Figures of the nasal and temporal angles (3 and 9 o'clock positions) were obtained according to the anatomical mark. At least three images were obtained for a single quadrant, the one with the clearest visibility of angle structures was chosen for further evaluation. As for SD-OCT, a CAM-L lens (cornea lens adapter; Optovue, Inc.) was mounted over the imaging aperture. The SD-OCT imaging was performed according to the CL Angle protocol (software version 4.0.7.5; RTVue OCT; Optovue, Inc., Fremont, CA). As for SSOCT, the Angle (HD) (software version 6A; SS-1000 CASIA, Tomey, Nagoya, Japan) protocol was used to capture images.

\section{Image analysis}

The following angle structures were of interest and identified:

1. Visualization of the SS: The SS in anterior segment imaging is marked by a prominent inner extension of the sclera and represents an anatomical landmark for the junction between the inner wall of the trabecular meshwork and the sclera [4].

2. Visualization of the SL: The SL is defined as the posterior limbal zone bordering the cornea where Descemet's membrane terminates [5].

3. Visualization of the SC: The SC is seen as a curvilinear lucent area external to the trabecular meshwork. This lucent area extended from the scleral spur to the anterior tip of the trabecular meshwork located at the end of the Descemet's membrane [6]. 

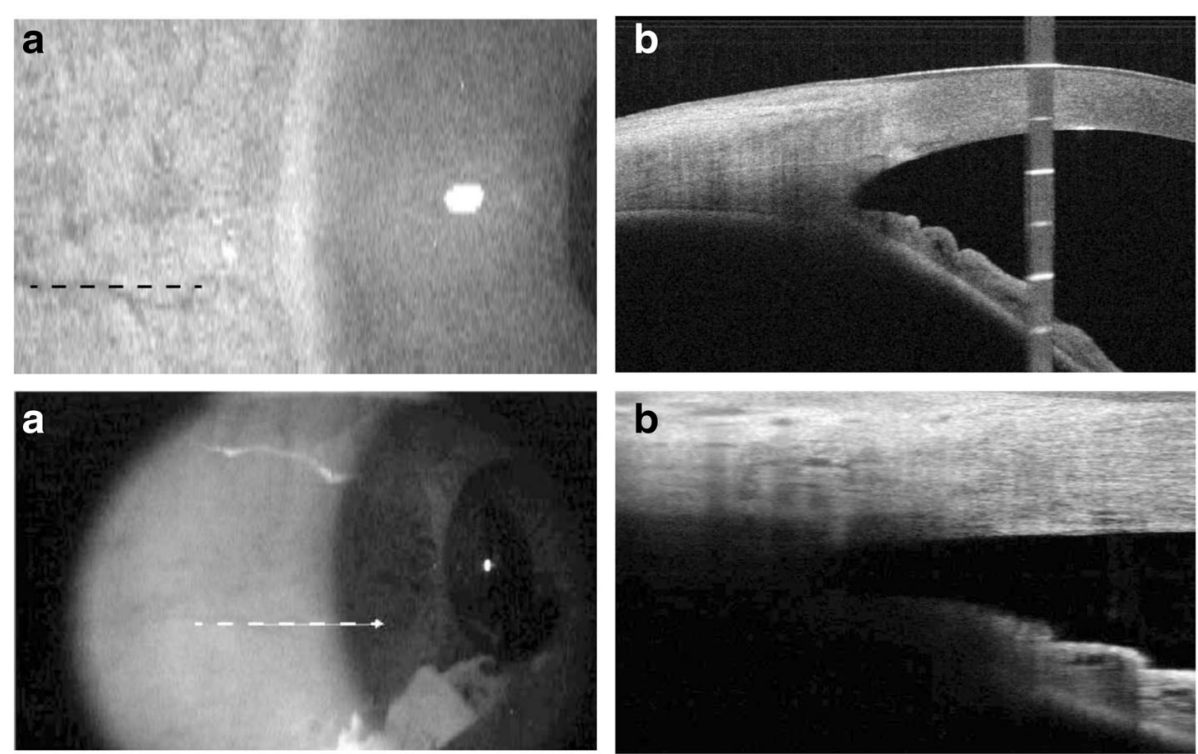

Fig. 2 OCT imaging of the same location of anterior chamber. When using SS-OCT, we firstly identified the vessel chosen in Fig. 1 as indicated by black dotted line (a), and then took the cross-sectional image (b). Similarly, the same vessel was located under SD-OCT as was outlined by white dotted line in (c) to make sure that the image (d) shows the same part of the anterior chamber

4. The LTM was defined as the distance from the Schwalbe's line to the scleral spur.

5. AOD500 is the distance between a point of the cornea which is $500 \mu \mathrm{m}$ away from the scleral spur and the opposite point of the iris [7].

6. AOD750, likewise, is the distance between a point of the cornea which is $750 \mu \mathrm{m}$ away from the scleral spur and the opposite point of the iris.

7. The LSC was defined as the distance from the highest to the lowest point measured from the cross-sectional image of the Schlemm's canal.

The nasal and temporal angles were both measured in this study. An illustration of identification and measurement of structures is depicted in Fig. 3 and Fig. 4. All OCT scans were analyzed separately by two examiners (JYC, YSQ) masked to clinical findings, and the interobserver reproducibility for the angle assessment was evaluated in a random selection of 30 images. The calculated intraclass correlation coefficient $(95 \% \mathrm{CI})$ was $0.98(0.97-0.99)$.

\section{Statistical analysis}

For the continuous variables, data obtained from two OCT scans were first examined by Kolmogorov-Smirnov test for the normality of distribution. For those variables which complied with normal distribution, paired Student's $\mathrm{t}$ test was used to compare the difference between measurements made by two OCTs and the disparity between nasal and temporal data measured by the same device. Otherwise, Wilcoxon rank-sum test was applied to do the comparison. The correlation and agreement between SS-OCT and SD-OCT were evaluated by Pearson correlation coefficients (or Spearman's rank correlation coefficient, based on the normality of distribution)
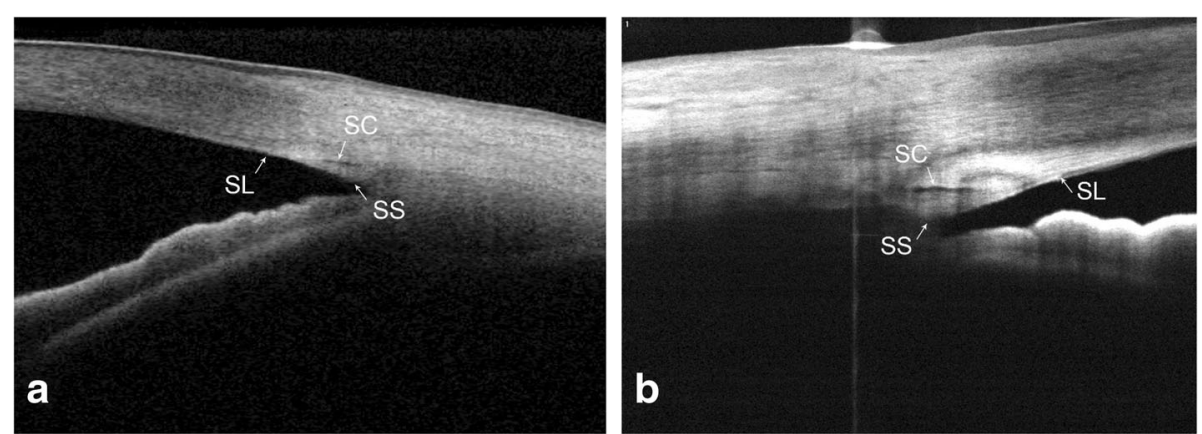

Fig. 3 Cross-sectional images of SS-OCT (a) and SD-OCT (b) Pictures of iridocorneal angle were obtained during OCT examination, and angle structures are demonstrated. (SC = Schlemm's canal; SS = scleral spur; SL = Schwalbe's line) 

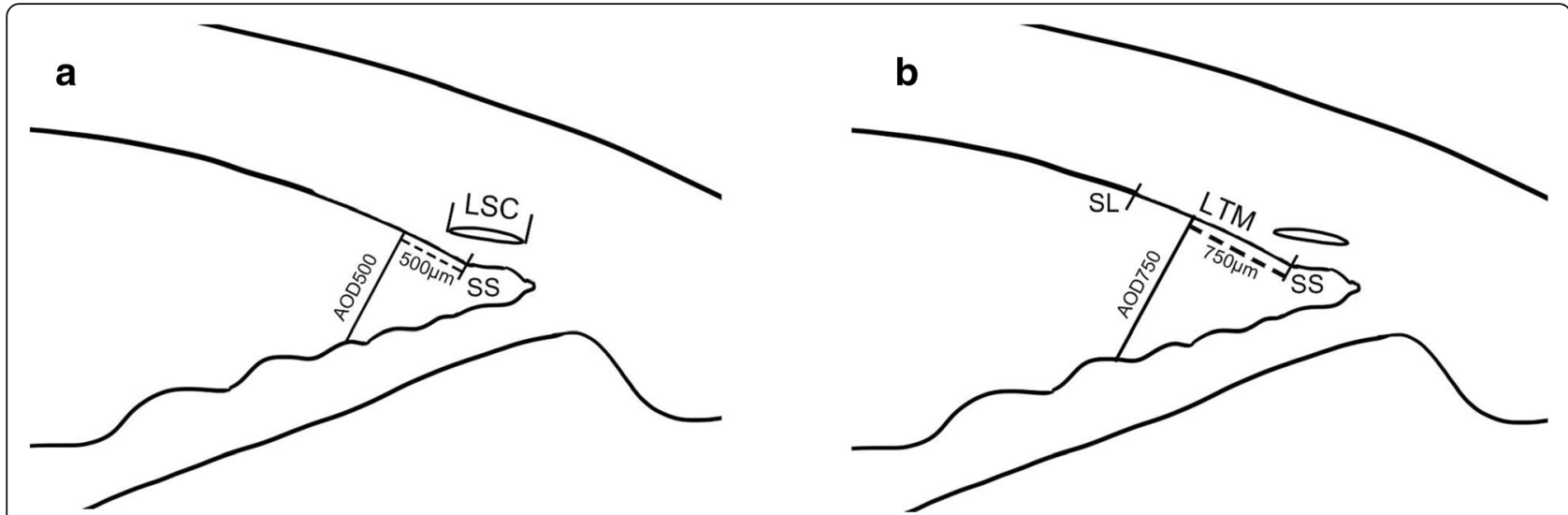

Fig. 4 The illustrations of angle measurement (LSC= the length of the Schlemm's canal; $L T M=$ the length of the trabecular meshwork; $A O D=$ angle opening distance; SS = scleral spur; SL = Schwalbe's line)

and Bland-Altman plots, respectively. As for the dichotomous variables, Fisher's exact test was adopted to analysis the measurements of two OCT scans. Statistical analysis were performed with SPSS software (version 24.0, IBM Corp.). A $P$ value less than 0.05 was considered statistically significant.

\section{Results}

The study comprised 67 eyes of 67 healthy subjects among which $37.3 \%$ (25) were male and $62.7 \%$ (42) were female. The mean \pm SD age was $60.98 \pm 7.76$ years.

\section{Identification of angle structures}

\section{SS-OCT}

Using SS-OCT OCT, the SS, SL, and SC were identified in 67(100\%)/67(100\%), 61(91.04\%)/58(86.57\%), and $34(50.75 \%) / 46(68.66 \%)$ of subjects at the nasal and temporal quadrants, respectively. (Table 1).

\section{$S D-O C T$}

Using SD-OCT OCT, the SS, SL, and SC were identified in 50(74.63\%)/44(65.67\%), 60(89.55\%)/61(91.04\%), and $27(40.30 \%) / 47(70.15 \%)$ of subjects at the nasal and temporal quadrants, respectively. (Table 1).

Table 1 Comparison of angle structures discerning ability between SS-OCT and SD-OCT

\begin{tabular}{|c|c|c|c|c|c|c|}
\hline \multirow[t]{2}{*}{ Structure } & & \multicolumn{2}{|l|}{ TOMEY } & \multicolumn{2}{|c|}{ OPTOVUE } & \multirow[t]{2}{*}{$P$ value } \\
\hline & & $N=67$ & $\%$ & $\mathrm{~N}=67$ & $\%$ & \\
\hline \multirow[t]{2}{*}{ SS } & $(\mathrm{N})$ & 67 & 100.00 & 50 & 74.63 & $<0.0000$ \\
\hline & $(\mathrm{T})$ & 67 & 100.00 & 44 & 65.67 & $<0.0000$ \\
\hline \multirow[t]{2}{*}{ SL } & $(\mathrm{N})$ & 61 & 91.04 & 60 & 89.55 & 1.0000 \\
\hline & $(\mathrm{T})$ & 58 & 86.57 & 61 & 91.04 & 0.6072 \\
\hline \multirow[t]{2}{*}{ SC } & $(\mathrm{N})$ & 34 & 50.75 & 27 & 40.30 & 0.2810 \\
\hline & $(\mathrm{T})$ & 46 & 68.66 & 47 & 70.15 & 1.0000 \\
\hline
\end{tabular}

SS Scleral spur, SL Schwalbe's line, SC Schlemm's canal
Visualization of the SS from both nasal and temporal quadrants was achieved by SS-OCT. Both devices presented satisfying detection rate for the SL. And the difference between the two OCTs for the same quadrant was not statistically significant $(p=1.0000$ for nasal side, $p=0.6072$ for temporal side), either as the two quadrants measured by the same device ( $p=0.5488$ for SSOCT, $\mathrm{p}=1.0000$ for SD-OCT). Whereas, the identification of the SC was not that fulfilling, and both OCTs made better performance in identifying the SC from temporal side than that from nasal side $(p=0.0290$ for SS-OCT, $p=0.0022$ for SD-OCT) .

\section{Measurement of angle structures \\ SS-OCT}

Using SS-OCT, the LTM, AOD500, AOD750 and the LSC were $702.49 \pm 108.32 \mu \mathrm{m} \quad / 669.84 \pm 100.21 \mu \mathrm{m}$, $283.64 \pm 128.56 \mu \mathrm{m} \quad / \quad 333.99 \pm 150.12 \mu \mathrm{m}, \quad 391.33 \pm$ $176.86 \mu \mathrm{m} / 462.30 \pm 216.61 \mu \mathrm{m}$, and $225.91 \pm 41.44 \mu \mathrm{m} /$ $243.85 \pm 43.34 \mu \mathrm{m}$ at the nasal and temporal quadrants, respectively. (Table 2).

\section{$S D-O C T$}

Using SD-OCT OCT, the LTM, AOD500, AOD750 and the LSC were $657.08 \pm 112.15 \mu \mathrm{m} / 648.67 \pm 101.82 \mu \mathrm{m}$, $314.98 \pm 148.52 \mu \mathrm{m} \quad / \quad 370.36 \pm 186.85 \mu \mathrm{m}, \quad 424.06 \pm$ $196.32 \mu \mathrm{m} / 491.93 \pm 255.04 \mu \mathrm{m}$, and $215.70 \pm 50.82 \mu \mathrm{m} /$ $215.83 \pm 37.99 \mu \mathrm{m}$ at the nasal and temporal quadrants, respectively. (Table 2).

Notably, there were significant difference in AOD500 $(p<0.000$ for nasal and temporal side) and AOD750 ( $\mathrm{p}<0.000$ for nasal and $p=0.009$ for temporal side) between SS-OCT and SD-OCT. Significant difference of AOD500 and AOD750 also existed between nasal and temporal sides regardless of the measuring instrument (all $\mathrm{p}<0.000$ ). Additionally, the LTM and LSC in the nasal side was longer when evaluated by SS-OCT ( $p=$ 
Table 2 Comparison of angle structures measurements between SS-OCT and SD-OCT

\begin{tabular}{|c|c|c|c|c|c|c|}
\hline & & TOMEY & OPTOVUE & $P$ value & Correlation & $P$ value \\
\hline LTM & $(\mathrm{N})$ & $702.49 \pm 108.32$ & $657.08 \pm 112.15$ & 0.002 & 0.510 & 0.000 \\
\hline$(\mu \mathrm{m})$ & $(T)$ & $669.84 \pm 100.21$ & $648.67 \pm 101.82$ & 0.137 & 0.418 & 0.009 \\
\hline AOD500 & $(\mathrm{N})$ & $283.64 \pm 128.56$ & $314.98 \pm 148.52$ & $0.000^{\mathrm{a}}$ & $0.897^{\mathrm{b}}$ & 0.000 \\
\hline$(\mu \mathrm{m})$ & (T) & $333.99 \pm 150.12$ & $370.36 \pm 186.85$ & $0.000^{\mathrm{a}}$ & $0.811^{b}$ & 0.000 \\
\hline AOD750 & $(\mathrm{N})$ & $391.33 \pm 176.86$ & $424.06 \pm 196.32$ & $0.000^{\mathrm{a}}$ & $0.927^{b}$ & 0.000 \\
\hline$(\mu \mathrm{m})$ & (T) & $462.30 \pm 216.61$ & $491.93 \pm 255.04$ & $0.009^{a}$ & $0.811^{\mathrm{b}}$ & 0.000 \\
\hline LSC & $(\mathrm{N})$ & $225.91 \pm 41.44$ & $215.70 \pm 50.82$ & 0.074 & 0.704 & 0.003 \\
\hline$(\mu \mathrm{m})$ & $(T)$ & $243.85 \pm 43.34$ & $215.83 \pm 37.99$ & 0.007 & 0.093 & 0.597 \\
\hline
\end{tabular}

LTM Length of trabecular meshwork, AOD500 Angle opening distance $500 \mu \mathrm{m}$, AOD750 Angle opening distance $750 \mu \mathrm{m}$, LSC Length of Schlemm's canal

${ }^{\text {a }}$ Wilcoxon rank-sum test

b Spearman's rank correlation coefficient

' This $P$ value is for correlation

$0.002, p=0.007$ respectively). A statistically significant good correlation was found between these two devices in all parameters except for the LSC in temporal side $(p=0.597, r=0.093)$. Nevertheless, SS-OCT and SDOCT had poor agreement in these parameters. The 95\% LoA for the nasal/temporal LTM, AOD500, AOD750 and LSC between these two devices were -154.0 to $257.1 / 188.3$ to $242.5 \mu \mathrm{m},-179.8$ to $90.5 /-238.4$ to $153.3 \mu \mathrm{m},-208.7$ to $100.1 /-299.7$ to $233.2 \mu \mathrm{m}$ and 52.5 to $88.2 /-78.7$ to $131.0 \mu \mathrm{m}$, respectively.

\section{Discussion}

The identification of the SS is crucial in angle assessment, as it offers a reference point of discerning trabecular meshwork and serves as a landmark for quantitative measurements such as AOD500/750 and the LTM [4]. Our study demonstrated impressive identification ability of the SS at nasal and temporal quadrants using SSOCT compared with SD-OCT. Since the central wavelength of SS-OCT is $1310 \mathrm{~nm}$, it is endowed with a powerful penetrability (6 $\mathrm{mm}$ in depth) to detect deeper structures. Satisfactory visualization of the SS using SSOCT was also achieved by the study of Cumba et al. [8]. Additionally, they reported good interobserver reproducibility of SS-OCT for SS identification, especially in temporal (87\%) and nasal (81\%) quadrants. Similarly, time-domain OCT with an equal central wavelength also presents an advantage of detecting the SS. In the study by Leung et al. [2] SS can be seen in 98.0\% (nasal) and $85.7 \%$ (temporal) of the subjects using slit-lamp OCT and in $98.0 \%$ (nasal) and $96.0 \%$ (temporal) of the subjects using Visante OCT. On the other hand, considering the employment of a superluminescent diode laser wavelength of $840 \mathrm{~nm}$, SD-OCT falls short of clear visibility of deep tissue structures such as the SS. However, it is possible for SD-OCT to identify more superficial structures such as the $\mathrm{SL}$, as we found in our study. Due to this feature, new methods of angle quantification with reference to the SL was proposed by Cheung et al. [9].

Opinions divide when it comes to the identification of the SC. Usui et al. [10] disagreed with Asrani et al. [11] concerning the morphology and location of the SC. The latter claimed that SC was an arched-shape black space that was located two thirds of the corneal thickness from the corneal surface at the limbus. Conversely, Usui et al. made an argument that analysis of OCT images of the angle structure was easily interfered with the coexistence of the cornea, sclera, SC, and trabecular meshwork which have different reflection and polarization properties [10]. According to their criteria of SC identification, $60.0 \%$ (nasal) and 63.3\% (temporal) of the SC in subjects' right eyes were completely observable. The same statistics for the fellow eyes were $90.0 \%$ (nasal) and $66.7 \%$ (temporal). We basically agreed their definition of the SC's morphology, whereas, their data were clearly inconsistent with the conclusion we drew. We attributed this disagreement to the different age range of tested subjects. Participants of our study were all above 50 years old whereas the subjects' age ranging from 29 to 81 in Usui's study. The transparency of cornea and sclera decreases as we age. For example, the development of pinguecula and pterygium could significantly interfere the visibility of underlying structures. (Fig. 5) In our study, there were no significant differences in discerning capability of the SC between SS-OCT and SD-OCT both at nasal and temporal quadrants $(p=0.2810, p=1.0000)$. Although, with a shorter wavelength, it should be more difficult to view the angle recess. However, Aung $\mathrm{T}$ et al. [12] reported good visualization of angle structures including the SC by SD-OCT with certain image processing. Considering the disparities among studies mentioned above, we hold that further studies should be dedicated to standardize the identification and measurement of the SC. 


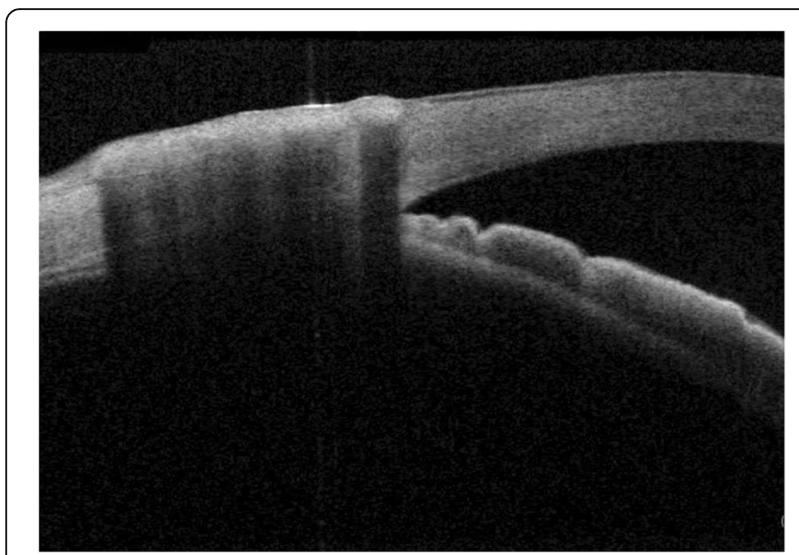

Fig. 5 Example of reduced scleral transparency caused by pinguecula

Our study demonstrated significant difference in the measurements of AOD500/750 between the two devices. For both OCTs, the temporal data were larger than the nasal ones. And for both quadrants, the measurements were larger using SD-OCT. The former finding could be supported by many other published results. Using slit lamp OCT and Visante OCT, Leung et al. reported that the nasal AOD500 were $534 \pm 234 \mu \mathrm{m} / 527 \pm 249 \mu \mathrm{m}$, while the temporal AOD500 were $628 \pm 254 \mu \mathrm{m} / 572 \pm$ $275 \mu \mathrm{m}$ [2]. Similar conclusions were also drawn by Pettersson et al. who measured the ACA in four meridians $\left(0^{\circ}, 94^{\circ}, 180^{\circ}, 274^{\circ}\right)$ with the Sirius Scheimpflug camera and found the mean nasal angle was $40.895 \pm 6.908$ degrees while the temporal $47.531 \pm 5.578$ degrees [13]. As to the latter conclusion, previous studies have been concentrated on the agreements between different instruments in angle quantification. Radhakrishnan et al. [14] showed that TD-OCT was similar to UBM in quantitative measurements of the angle such as AOD500 and Trabecular-iris space area (TISA) 500. Pan et al. [15] and Akil et al. [16] demonstrated in their study that SD-OCT was able to give consistent Schwalbe's line-based angle metrics. However, the studies on SS-OCT were relatively limited. In our study, although good correlation of the results between SS-OCT and SD-OCT was found, the analysis of Bland-Altman plots (Fig. 6 and Fig. 7) revealed that the two devices had poor agreements. The spans of $95 \%$ limits of agreement for the nasal/temporal LTM, AOD500, AOD750 and LSC between these two devices were 411.1/ $54.2 \mu \mathrm{m}, \quad 270.3 / 391.7 \mu \mathrm{m}, \quad 308.8 / 532.9 \mu \mathrm{m}$ and $140.7 /$ $209.7 \mu \mathrm{m}$, respectively. Considering different types of OCTs were compared in this study, some plausible postulations might serve to explain the differences. Firstly, it should be noted that the refraction of light at the anterior and posterior surface of the cornea leads to the distortion of angle measurements. Both OCT devices adopted a "dewarping" algorithm for the correction of these distortion, so the difference in algorithm (e.g. refractive indexes) should be considered. Secondly, since both devices use external target lights, the difference in illumination might contribute to the phenomenon. Unfortunately, after consulting with the manufacturers' representatives, we still couldn't get the exact illumination for the two instruments. Additionally, the distance between the light source and the tested eye could induce disparities in accommodation state, which could affect the lens position and the pupil size.

There were some limitations to this study. Firstly, the participants were all healthy subjects with normal angle conditions. The discerning ability of angle structures under ocular pathologies by different OCT devices was beyond our concern, which confined the extension of the conclusions. Secondly, the subjects were all above 50 years old. It remains to be verified whether the same conclusions can be drawn from younger populations. Thirdly, although the exact brightness of the external fixation lights of two OCT instruments was gauged, it's still unclear to what extent the measurement of ACA was effected. Since the fixation light directed the eyes of subjects to tilt for a certain angle so as to gain optimal
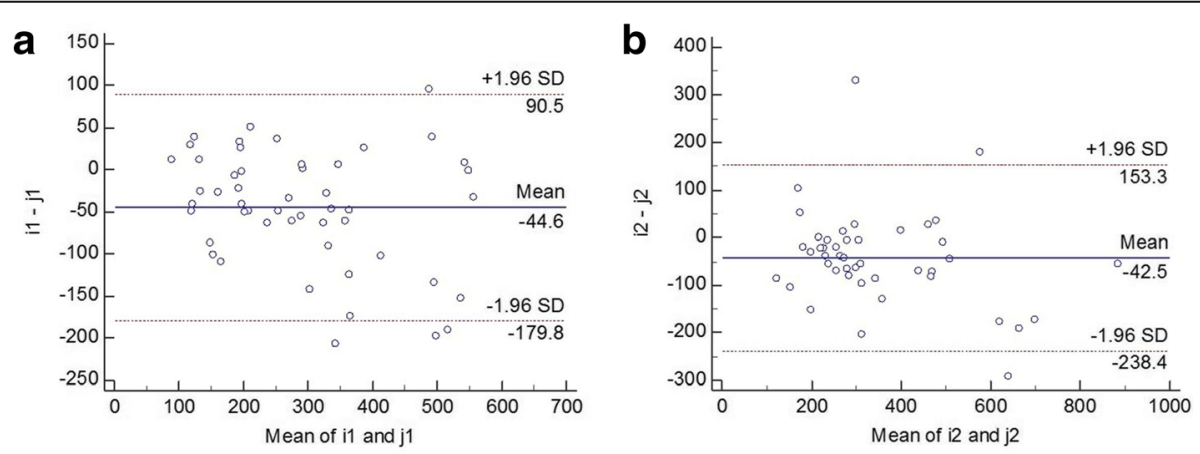

Fig. 6 Bland-Altman plots of AOD500 difference between SS-OCT and SD-OCT. a demonstrates the nasal quadrant and $\mathbf{b}$ demonstrates the temporal quadrants. (i1: AOD500 measured by SS-OCT nasally; i2: AOD500 measured by SS-OCT temporally; j1: AOD500 measured by SD-OCT nasally; j2: AOD500 measured by SD-OCT temporally) 

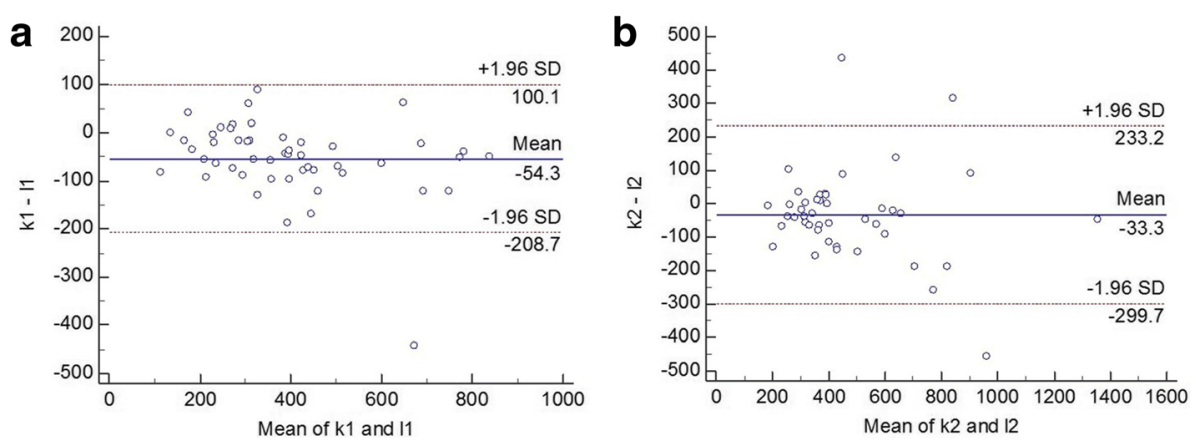

Fig. 7 Bland-Altman plots of AOD750 difference between SS-OCT and SD-OCT. a demonstrates the nasal quadrant and $\mathbf{b}$ demonstrates the temporal quadrants. (k1: AOD750 measured by SS-OCT nasally; k2: AOD750 measured by SS-OCT temporally; 11: AOD750 measured by SD-OCT nasally; I2: AOD750 measured by SD-OCT temporally)

visualization of temporal or nasal anterior chamber, the diameter of pupils remained unmeasurable.

Since their first introduction to the assessment of angle structures, different generations of AS-OCTs are now commercially available. Preceding studies have been conducted focusing on the measurement of the ACA. The current study comprising a relatively large number of consecutive patients and offered a new perspective about the value of SD-OCT and SS-OCT when it comes to angle evaluation.

\section{Conclusions}

In conclusion, we compared the qualitative and quantitative measurements obtained from SD-OCT (RTVue, Optovue Corporation, Fremont, CA) and SS-OCT (SS1000 CASIA, Tomey, Nagoya, Japan) in Chinese population. SS-1000 CASIA, as the representative to SS-OCT with longer central wavelength demonstrated excellent visualization of the SS, the landmark and reference point for angle measurement. However, the two devices barely distinguished itself from each other as to the identification of the SL and the SC. In the case of the SC, we found that the detection rate was higher at the temporal quadrant compared to the opposite side, regardless of the type of AS-OCT. Further optimization of the SC morphology under OCT scanning might contribute to the standardization of clinical findings. The measurement of angle (AOD500/750) showed significant difference between the two methods and the two quadrants. The poor agreements between SS-OCT and SD-OCT indicated that the data measured from these devices were not interchangeable, although good correlation of the results between the two devices was found. For clinicians and researchers, it is recommended that choices between different OCTs are made based on individual requirement. For example, SS-OCT displayed a better performance in detecting deeper structures. So one might prefer SS-OCT to SD-OCT when examining the scleral spur.

\section{Abbreviations}

ACA: Anterior chamber angle; AOD: Angle opening distance; ASOCT: Anterior segment optical coherence tomography; FD-OCT: Fourierdomain optical coherence tomography; LoA: Limits of agreement; LSC: the length of the Schlemnn's canal; LTM: The length of the trabecular meshwork; SC: The Schlemm's canal; SD-OCT: Spectrum domain optical coherence tomography; SL: The Schwalbe's line; SS: The scleral spur; SS-OCT: Swept source optical coherence tomography; TD-OCT: Time-domain optical coherence tomography; UBM: Ultrasound biomicroscopy

\section{Acknowledgements}

Not applicable.

\section{Authors' contributions}

Data analysis, writing paper, preparing figures and Tables Y.Q., data analysis C.T., data analysis M.Z., designing study X.S., data collection J.C. All authors read and approved the final manuscript.

\section{Funding}

This work was supported by Grants 81870661 and 81470623 from the National Natural Science Foundation of China. The funding organization had no role in the design or conduct of this research.

Availability of data and materials

All data included in this study are available upon reasonable request by contact with the corresponding author.

\section{Ethics approval and consent to participate}

The study was approved by the Human Research Ethics Committee of the Eye and ENT Hospital of Fudan University and adhered to the tenets of the Declaration of Helsinki. Written informed consent was obtained from each subject.

Consent for publication

Not applicable.

\section{Competing interests}

The authors declare that they no competing interests.

\section{Author details}

${ }^{1}$ Department of Ophthalmology \& Visual Science, Eye and ENT Hospital of Fudan University, 83 Fenyang Rd, Shanghai 200031, China. ${ }^{2}$ State Key Laboratory of Medical Neurobiology, Institutes of Brain Science and Collaborative Innovation Center for Brain Science, Fudan University, Shanghai 200032, China. ${ }^{3} \mathrm{NHC}$ Key Laboratory of Myopia (Fudan University), Key Laboratory of Myopia, Chinese Academy of Medical Sciences and Key Laboratory of Visual Impairment and Restoration of Shanghai, Shanghai 200031, China. 
Received: 9 April 2019 Accepted: 13 June 2019

Published online: 08 July 2019

\section{References}

1. Huang D, Swanson EA, Lin CP, Schuman JS, Stinson WG, Chang W, Hee MR, Flotte T, Gregory K, Puliafito CA, et al. Optical coherence tomography. Science. 1991;254(5035):1178-81.

2. Leung CK, Li H, Weinreb RN, Liu J, Cheung CY, Lai RY, Pang CP, Lam DS. Anterior chamber angle measurement with anterior segment optical coherence tomography: a comparison between slit lamp OCT and Visante OCT. Invest Ophthalmol Vis Sci. 2008:49(8):3469-74.

3. Miller AR, Roisman L, Zhang Q, Zheng F, Rafael de Oliveira Dias J, Yehoshua Z, Schaal KB, Feuer W, Gregori G, Chu Z, et al. Comparison between spectral-domain and swept-source optical coherence tomography angiographic imaging of choroidal neovascularization. Invest Ophthalmol Vis Sci. 2017:58(3):1499-505.

4. Sakata LM, Lavanya R, Friedman DS, Aung HT, Seah SK, Foster PJ, Aung T. Assessment of the scleral spur in anterior segment optical coherence tomography images. Arch Ophthalmol. 2008;126(2):181-5.

5. Breazzano MP, Fikhman M, Abraham JL, Barker-Griffith AE. Analysis of Schwalbe's line (Limbal smooth zone) by scanning Electron microscopy and optical coherence tomography in human eye Bank eyes. J Ophthalmic Vision Res. 2013;8(1):9-16.

6. Quek DT, Narayanaswamy AK, Tun TA, Htoon HM, Baskaran M, Perera SA Aung T. Comparison of two spectral domain optical coherence tomography devices for angle-closure assessment. Invest Ophthalmol Vis Sci. 2012;53(9): 5131-6.

7. Koprowski R, Wrobel Z, Wilczynski S, Nowinska A, Wylegala E. Methods of measuring the iridocorneal angle in tomographic images of the anterior segment of the eye. Biomed Eng Online. 2013;12:40.

8. Cumba RJ, Radhakrishnan S, Bell NP, Nagi KS, Chuang AZ, Lin SC, Mankiewicz KA, Feldman RM. Reproducibility of scleral spur identification and angle measurements using fourier domain anterior segment optical coherence tomography. J Ophthalmol. 2012;2012:487309.

9. Cheung CY, Zheng C, Ho CL, Tun TA, Kumar RS, Sayyad FE, Wong TY, Aung T. Novel anterior-chamber angle measurements by high-definition optical coherence tomography using the Schwalbe line as the landmark. $\mathrm{Br}$ Ophthalmol. 2011;95(7):955-9.

10. Usui T, Tomidokoro A, Mishima K, Mataki N, Mayama C, Honda N, Amano S, Araie M. Identification of Schlemm's canal and its surrounding tissues by anterior segment fourier domain optical coherence tomography. Invest Ophthalmol Vis Sci. 2011;52(9):6934-9.

11. Asrani S, Sarunic M, Santiago C, Izatt J. Detailed visualization of the anterior segment using fourier-domain optical coherence tomography. Arch Ophthalmol. 2008;126(6):765-71.

12. Kagemann L, Wollstein G, Ishikawa H, Bilonick RA, Brennen PM, Folio LS, Gabriele ML, Schuman JS. Identification and assessment of Schlemm's canal by spectral-domain optical coherence tomography. Invest Ophthalmol Vis Sci. 2010;51(8):4054-9.

13. Pettersson L, Källmark F. Difference in the anterior chamber angle of the four meridians. J Appl Med Sci. 2012;1(1):1-13.

14. Radhakrishnan S, Goldsmith J, Huang D, Westphal V, Dueker DK, Rollins AM, Izatt JA, Smith SD. Comparison of optical coherence tomography and ultrasound biomicroscopy for detection of narrow anterior chamber angles. Arch Ophthalmol. 2005:123(8):1053-9.

15. Pan X, Marion K, Maram J, Zhang ZY, Francis BA, Nittala MG, Sadda SR Chopra $\mathrm{V}$. Reproducibility of anterior segment angle metrics measurements derived from cirrus spectral domain optical coherence tomography. J Glaucoma. 2015:24(5):e47-51.

16. Akil H, Dastiridou A, Marion K, Francis B, Chopra V. Repeatability, reproducibility, agreement characteristics of 2 SD-OCT devices for anterior chamber angle measurements. Can J Ophthalmol. 2017;52(2):166-70.

\section{Publisher's Note}

Springer Nature remains neutral with regard to jurisdictional claims in published maps and institutional affiliations.

Ready to submit your research? Choose BMC and benefit from:

- fast, convenient online submission

- thorough peer review by experienced researchers in your field

- rapid publication on acceptance

- support for research data, including large and complex data types

- gold Open Access which fosters wider collaboration and increased citations

- maximum visibility for your research: over $100 \mathrm{M}$ website views per year

At BMC, research is always in progress.

Learn more biomedcentral.com/submissions 